\title{
Weight gain measured at 6 weeks after birth as a predictor for severe retinopathy of prematurity: study with 317 very low birth weight preterm babies
}

\author{
Joao Borges Fortes Filho • Pedro P. Bonomo • \\ Mauricio Maia - Renato S. Procianoy
}

Received: 18 August 2008 /Revised: 10 November 2008/Accepted: 11 November 2008 / Published online: 4 December 2008

(C) The Author(s) 2008. This article is published with open access at Springerlink.com

\begin{abstract}
Background Recent studies suggest that postnatal weight gain can play an important role in the development of retinopathy of prematurity (ROP).

Aim To analyze the low weight gain (WG) from birth to 6 weeks of life to predict the development of severe retinopathy of prematurity (ROP) among very low birth weight preterm babies (VLBW).

Methods A prospective cohort study included 317 newborns with birth weight (BW) $\leq 1,500 \mathrm{~g}$ and gestational age (GA) $\leq 32$ weeks. The main outcome was the development of severe ROP (defined as threshold ROP and higher stages of ROP). In all patients, the proportion of the WG was defined as the preterm weight measured at 6 weeks of life minus the BW divided by the BW. Seventeen risk factors for ROP were studied by univariate analysis. Chi-square test and Student's $t$-test were used to compare no-ROP/mild ROP patients and severe ROP patients. Logistic regression and receiver operating charac-
\end{abstract}

\section{J. B. F. Filho $(\square)$}

Medical School, Department of Ophthalmology,

Federal University of Rio Grande do Sul and Hospital de Clínicas de Porto Alegre,

Rua Ramiro Barcelos, 2350,

Porto Alegre, RS 90035-003, Brazil

e-mail: jbfortes@cursohbo.com.br

\section{R. S. Procianoy}

Department of Pediatrics, Newborn Section,

Federal University of Rio Grande do Sul and Hospital de Clinicas de Porto Alegre,

Porto Alegre, Brazil

\section{P. P. Bonomo $\cdot$ M. Maia}

Medical School, Department of Ophthalmology,

Federal University of Sao Paulo,

Sao Paulo, Brazil teristic (ROC) curve were used to determine if the WG proportion was independently related to severe ROP development and if it was capable of predicting severe ROP. Ophthalmological examinations started between the fourth and sixth week of life, and were repeated until the 45th week of postmenstrual age. Weight gain proportion was always calculated at completed 6 weeks of life.

Results Mean GA and mean BW of the whole cohort were 29.6 weeks $( \pm 1.9)$ and 1,124 grams $( \pm 239.5)$ respectively. After logistic regression, the low WG proportion under $51.2 \%$ from the BW, measured at 6 weeks of life, showed OR 3.007 (95\%CI: 1.195-7.566; $P=0.019$ ), for severe ROP, when adjusted for $\mathrm{BW}$ and for any stage intraventricular hemorrhage. Area under the ROC curve was 0.63 (95\%CI: $0.495-0.761 ; P=0.037)$. For the discriminative cutoff of $51.2 \%$ of the WG proportion, sensitivity and specificity values were $66.3 \%$ and $62.6 \%$ respectively. Positive and negative predictive values were $10.2 \%$ and $94.7 \%$ respectively.

Conclusions Low WG by six weeks of life is an important and independent risk factor for severe ROP and is capable to predict the development of severe ROP in most patients that needed treatment.

Keywords Retinopathy of prematurity · Risk factors . Weight gain · Prevalence $\cdot$ VLBW infants

\section{Introduction}

Retinopathy of prematurity (ROP) is an important complication of preterm newborns. ROP was first described in 1942, and quickly became a leading cause of childhood blindness in industrialized countries [1]. Recently, ROP has been also identified as one of the most important causes of treatable blindness among children in many countries in 
Latin America, China and India and among the former emerging economies from East Europe, due to the increase in the survival rates among preterm newborns $[2,3]$.

ROP is a multifactorial disease, and the main postnatal risk factors described are oxygen-therapy, low gestational age (GA), low birth weight (BW), sepsis, intraventricular hemorrhage, blood transfusion, and use of indomethacin, surfactant and erythropoietin [4-6].

Postnatal low weight gain (WG) of very low birth weight (VLBW) infants is a recently mentioned risk factor for ROP [7-9]. Only three previous studies, a retrospective [7], a case-control [8], and a prospective cohort [9], reported the association between postnatal low WG and occurrence of ROP in VLBW premature infants. In the last of these studies, Löfqvist et al. [9] demonstrated the usefulness of the longitudinal postnatal WG and serum insulin-like growth factor-I (IGF-I) measurements from birth until postmenstrual age of 36 weeks to predict ROP. There is no prospective study addressing only the importance of the VLBW infants' WG and the occurrence of severe ROP. In the year 2002, we designed a prospective cohort study to analyze if the WG from birth to 6 weeks of life could be an independent risk factor for severe ROP and if it could predict severe ROP, once the low postnatal WG is easily identifiable as a risk factor, during the screening sessions to detect ROP.

\section{Methods}

\section{Patients}

We performed a prospective cohort study analyzing the prevalence of severe ROP from the initial ophthalmologic examination up to the 45th week of postmenstrual age and the WG proportion from birth up to 6 weeks of life. All preterm neonates admitted to the Neonatal Intensive Care Unit (NICU) of Hospital de Clínicas de Porto Alegre, Brazil from October 2002 to November 2007, with BW $\leq 1,500$ grams and GA $\leq 32$ weeks, were included. Infants that died before completing 6 weeks of life or before 45 weeks of postmenstrual age were excluded. No other exclusion criteria were used. The ophthalmic examination was always performed by the same author (JBFF) and consisted of binocular indirect ophthalmoscopy with a 28 D Nikon lens (Nikon ${ }^{\circledR}$, Melville, NY, USA) and the lid speculum for newborn (Alfonso Eye Speculum, Storz ${ }^{\circledR}$, Bausch \& Lomb Inc., San Dimas, CA, USA), after dilation of the pupils with eye drops containing tropicamide $0.5 \%$ and phenylephrine $2.5 \%$. Scleral indentation was used in order to achieve a better examination of the peripheral zone III.

According to the Brazilian guidelines for ROP screening, all infants were first examined between 4 and 6 weeks of life and then followed once or twice a week depending on the severity of the disease or until retinal vascularization was completed. All babies were allways examined in the NICU while hospitalized, and as outpatients after discharge up to completed 45 weeks of postmenstrual age.

\section{Outcome and variables}

The main clinical outcome was the occurrence of severe ROP in either eye during the entire observational period. The worst ROP stage, in either eye, was always recorded, according to the International Classification of 1984/1987 $[10,11]$. Severe ROP was defined as any ROP stage 3, threshold disease, or more. Threshold disease was defined according to the Multicenter Trial of Cryotherapy for ROP [12]. The prevalence of ROP was calculated with $95 \%$ confidence intervals.

The main variable was the WG proportion measured at completed 6 weeks of life in relation to BW. It was calculated as the baby's weight measured at 6 weeks of life minus the BW, divided by the BW, in all patients. Perinatal variables considered for the study were the already described risk factors for ROP: BW, GA, gender, use of oxygen-therapy on mechanical ventilation or on nasal Continuous positive airway pressure (CPAP), single or multiple gestation, use of surfactant, indomethacin and erythropoietin therapies, Apgar score at 5 minutes, sepsis, meningitis, all intraventricular hemorrhage stages, blood transfusions, persistent ductus arteriosus, weight at 6 weeks of life, and the absolute WG at 6 weeks of life (defined as the baby's weight at completed 6 weeks of life minus the $\mathrm{BW}$ ). Those data were obtained prospectively.

\section{Statistical methods and ethics}

The chi-square test was used to compare no-ROP/mild ROP patients and severe ROP patients. Student's unpaired $t$-test was used to compare continuous data. In the logistic regression, the dependent variables were chosen by their significance after univariate analysis. The main variable (WG proportion from birth to the sixth week of life) was included in the logistic regression as a continuous variable, without cutoff points, as well as it was included with the best discriminative sensibility/specificity value after the ROC curve results as a cutoff point. Patients under this cutoff point were classified as low WG patients. Odds ratio (OR) was calculated and compared after adjustments in both situations. Confidence interval $95 \%$ and significance level of $P<0.05$ were recorded. Receiver operating characteristic (ROC) curve was calculated for the proportional WG and severe ROP development. Statistical analysis was performed using the Statistical Package for Social Sciences software (SPSS 14.0 for Windows, SPSS Inc., Chicago, IL, 
USA). The study protocol was approved by the Ethics Committee of the HCPA.

\section{Results}

The prospective cohort comprised 317 neonates, of whom $183(57.7 \%)$ were female and $63.7 \%$ were small for GA (SGA $<10$ percentile) patients. Birth weight in all studied newborns ranged from 505 to 1,500 grams, with a mean of 1,124 grams (SD 239.5). Gestational age ranged from 24 to 32 weeks, with a mean of 29.7 weeks (SD 1.9). Severe ROP needing treatment occurred in 24 patients (7.2\%), among whom 23 achieved ROP stage 3, threshold disease and were treated by transpupillary diode laser photocoagulation. One patient reached ROP stage 4 despite two laser treatments. Only one patient reached ROP stage $5(0.3 \%)$ by missing the scheduled treatment after NICU discharge, and only one baby, born at 32 weeks GA and with BW of 1,315 grams, developed ROP (threshold disease) at postmenstrual age of 41 weeks. This baby achieved 2,200 grams by the sixth week of life (with $68 \%$ of the WG proportion, as defined). The mean GA for pre-threshold ROP among our patients was 36.3 (SD 1.6) weeks and for threshold ROP was 37.9 (SD 1.7) weeks. The demographic characteristics of all included patients, as well as the complete prevalence of ROP, are displayed in Table 1.

In Table 2 we show the univariate comparison between no-ROP/mild ROP and severe ROP patients: BW $(P<$ $0.001)$, GA $(P<0.001)$, weight at 6 weeks of life $(P<$ $0.001)$, WG $(P<0.001)$, WG proportion $(P=0.019)$, and

Table 1 Prevalence of ROP and demographic characteristics of the study population

\begin{tabular}{ll}
\hline Characteristic & Value \\
\hline Number of patients & 317 \\
No-ROP & $219(69.1 \%)$ \\
ROP & $98(30.9 \%)$ \\
ROP 1 & $42(13.2 \%)$ \\
ROP 2 & $32(10.1 \%)$ \\
ROP 3* & $22(6.9 \%)$ \\
ROP 4* & $1(0.3 \%)$ \\
ROP 5* & $1(0.3 \%)$ \\
Female gender & $183(57.7 \%)$ \\
SGA & $202(63.7 \%)$ \\
Mean BW (grams) $)^{* *}$ & $1,124.0 \pm 239.5$ \\
Mean GA (weeks) & $* *$ \\
Mean WG (grams) & $29.6 \pm 1.9$ \\
\hline
\end{tabular}

*: Severe ROP needing treatment; **: Data presented as mean \pm standard deviation; SGA: small for the gestational age $(<10$ percentile); BW: birth weight; GA: gestational age; WG: weight gain from birth to the sixth week of life. any stage intraventricular hemorrhage $(P=0.047)$ were significantly lower in severe ROP patients.

After logistic regression, the WG proportion from birth to the sixth week of life, adjusted for BW and for any stage intraventricular hemorrhage, showed OR 3.007 (95\%CI: $1.195-7.566 ; P=0.019)$ when considered the cutoff point under $51.2 \%$ of the $\mathrm{BW}$; and as a continuous variable, without cutoff points, it showed OR 1.032 (95\%CI: 1.008$1.055 ; P=0.008$ ), Table 3 .

For severe ROP, the area under the ROC curve was 0.63 (95\%CI: $0.495-0.761 ; P=0.037$ ). For the best discriminative cutoff of $51.2 \%$ of the proportional WG over the BW, sensitivity and specificity values were $66.3 \%$ and $62.6 \%$ respectively. Positive and negative predictive values were $10.2 \%$ (95\%CI: $6.1-15.9 \%)$ and $94.7 \%$ (95\%CI: 90.5-97.4\%) respectively.

\section{Discussion}

Our study showed that WG proportion under $51.2 \%$ from birth to 6 weeks of life was an important and independent risk factor for severe ROP, and was capable of predicting the development of severe ROP needing treatment in the group of VLBW preterm babies. Our results are also in accordance with the longitudinal growth curves for hospitalized VLBW described by Ehrenkranz et al. [13] in 1999, and widely used in modern neonatology, in which these patients are expected to regain weight, around $50 \%$ of their $\mathrm{BW}$, after completed 6 weeks of life.

ROP pathogenesis is still incompletely understood. For many years, the high oxygen levels administered to neonates was considered the main risk factor for ROP development [14]; however, the disease kept occurring even after careful control in oxygen administration [15]. To date, no absolutely safe level for oxygen use has been demonstrated $[16,17]$. In our institution, oxygen administration is always monitored by pulse oxymetry, with recommended saturation between $88-94 \%$. The overall incidence of ROP in our study was similar to other published results [18-20].

The presence of any stage intraventricular hemorrhage was significant as a risk factor for ROP in our cohort of patients. Procianoy et al. [21], in 1981, related a significant association between cicatricial ROP and intraventricular hemorrhage in 138 VLBW. Christiansen et al. [22], in 2002, published a significant association between intraventricular hemorrhage and ROP in $60 \mathrm{VLBW}$ where neonates with more severe intraventricular hemorrhage grades reached severe ROP.

Low BW and GA have been implicated most directly in ROP development [23-25], but low WG as an independent risk factor for ROP development was just recently 
Table 2 Univariate analysis of the risk factors for the development of severe ROP

*Data presented in mean \pm standard deviation (Student's $t$-test); BW: birth weight; nasal CPAP: nasal continuous positive airway pressure

\begin{tabular}{llll}
\hline & $\begin{array}{l}\text { No-ROP/Mild-ROP patients } \\
(n=293)\end{array}$ & $\begin{array}{l}\text { Severe ROP patients } \\
(n=24)\end{array}$ & $P$ \\
\hline Birth weight (grams) ${ }^{*}$ & $1,141.6 \pm 231.7$ & $908.7 \pm 232.6$ & $<0.001$ \\
Gestational age (weeks) & $29.8 \pm 1.8$ & $27.9 \pm 2.2$ & $<0.001$ \\
Weight at 6 weeks of life (grams) ${ }^{*}$ & $1,753.9 \pm 362.5$ & $1,319.2 \pm 419.7$ & $<0.001$ \\
Weight gain (grams) ${ }^{*}$ & $612.3 \pm 205.6$ & $410.4 \pm 279.1$ & $<0.001$ \\
Weight gain proportion (\%) & $54.6 \pm 18.3$ & $45,1 \pm 25.1$ & 0.019 \\
Apgar score at 5 minutes* & $7.8 \pm 1.7$ & $7.6 \pm 1.7$ & 0.653 \\
Use of oxygen on mechanical ventilation & $152(52.1 \%)$ & $15(62.5 \%)$ & 0.397 \\
Use of oxygen on nasal CPAP & $230(78.5 \%)$ & $20(83.3 \%)$ & 0.795 \\
Use of erythropoietin & $231(79.1 \%)$ & $20(83.3 \%)$ & 0.819 \\
Use of indomethacin & $100(34.1 \%)$ & $9(37.5 \%)$ & 0.824 \\
Any intraventricular hemorrhage stage & $45(15.4 \%)$ & $8(33.3 \%)$ & 0.047 \\
Blood transfusions & $129(44.0 \%)$ & $14(58.3 \%)$ & 0.204 \\
Use of surfactant & $140(47.8 \%)$ & $14(58.3 \%)$ & 0.397 \\
Multiple gestation & $46(15.7 \%)$ & $4(16.7 \%)$ & 1.000 \\
Sepsis & $200(68.3 \%)$ & $19(79.2 \%)$ & 0.360 \\
Meningitis & $19(6.5 \%)$ & $3(12.5 \%)$ & 0.486 \\
Persistent ductus arteriosus & $40(13.7 \%)$ & $3(12.5 \%)$ & 1.000 \\
Female gender & $170(58.0 \%)$ & $13(54.2 \%)$ & 0.830 \\
& & &
\end{tabular}

described. Hall et al. [26], in 1995, related a low WG associated with severity of ROP in four survivors in a birth of quintuples with identical GA and similar BW. Homes and Düffner [27], in 1996, demonstrated that rats submitted to growth retardation developed retinal neovascularization more frequently and more severely.

A retrospective study by Wallace et al. [7], published in 2000, analyzed postnatal WG and another 11 risk factors in a group of 111 neonates. They suggested that WG under $50 \%$ of $\mathrm{BW}$ at 6 weeks of life indicated an important risk for severe forms of ROP. According to Wallace et al., the study results could not be explained by the difference in the average BW among the patients with severe ROP and those with less severe or no ROP, but by the difference in WG proportion in the 6 -week period after premature birth, and they concluded that there was a significant difference in WG in infants with severe and discrete or no ROP. Shaffer et al. [28] previously claimed that the absolute ratio of $\mathrm{WG}$ in VLBW would be proportional to the neonate's BW, and consequently, the expected proportion of WG should be the same for preterm infants with different BW in the same period of life.

Allegaert et al. [8], in 2003, in a prospective case-control study registered perinatal growth characteristics of 31 neonates with ROP threshold disease and in 31 neonates with the same GA who didn't develop ROP. They concluded that small for GA preterm newborns and those with intrauterine growth restriction had an increased risk of developing ROP threshold disease. In contrast, the relative WG (grams/kg/day) was not significantly different in the group with ROP and in the control group.

Recently, some studies showed the relation between the insulin-like growth factor-I (IGF-I) and ROP development. Hellström et al. [29] reported that in preterm infants with the same GA, IGF-I serum levels during the postnatal period were significantly and proportionally lower in those that developed ROP than in the ones who did not. In 2006 , Löfqvist et al. [9] showed that weekly measurements of postnatal WG together with a serum level of IGF-I were a useful marker for the risk of severe ROP. In this article, the authors disclosed some very important conclusions indicating the need to monitor the postnatal factors of weight, IGF-I level and IGF binding protein 3 level in order to enhance the clinician's ability to identify patients who will require treatment for ROP. Unfortunately, this approach seems to have some difficulties of use among ophthalmologists during the screening sessions to detect ROP, mainly in the middle-income countries context; once-weekly

Table 3 Odds ratio to develop severe ROP after logistic regression adjusted when a weight gain proportion is used as a continuous variable, and the weight gain proportion under $51.2 \%$ of the birth weight, according to the ROC curve

\begin{tabular}{llll}
\hline & OR & $95 \%$ CI & $P$ \\
\hline Continuous WG proportion (without cutoffs)* & 1.032 & $1.008-1.055$ & 0.008 \\
Low WG proportion* (WG $<51.2 \%$ of the BW) & 3.007 & $1.195-7.566$ & 0.019 \\
\hline
\end{tabular}

WG: weight gain; *: adjusted for birth weight (BW) and for any stage intraventricular hemorrhage. 
laboratory measurements of IGF-I and IGF binding protein 3 serum levels are cost expensive for national health care systems, and also, these laboratory tools are not widely available in most of the Latin American countries.

Engström et al. [30] related the role of maternal factors, postnatal nutrition and the WG in regulation of serum IGF-I among preterm infants. IGF-I is present in the natural maternal milk, and so an adequate natural breast-feeding could help in the prevention of ROP.

A question beyond the scope of our study is about why these babies are poor at gaining weight. The most practical answer to this question could be because they are sicker babies; but once we know about low $\mathrm{WG}$ as a risk factor for ROP, it is possible to follow-up these babies closely before the development of treatable ROP, trying to improve their clinical conditions. According to Ehrenkranz et al. [13], the WG after the second week of life is related to the nutritional support, and healthier babies usually gain weight faster than sicker babies. The low WG in the postnatal period could be indicative for the development of general morbidities, some of them also related with ROP development, such as sepsis, intraventricular hemorrhage, bronchopulmonary dysplasia, or necrotizing enterocolitis.

Our study aimed to prospectively analyze a cohort of patients with an adequate number of VLBW infants to determine if the proportion of postnatal WG is a risk factor for ROP development. After logistic regression, our results showed that WG proportion lower than $51.2 \%$, according to the ROC curve, was an independent risk factor for severe ROP development. Furthermore, our study showed that all of the three variables related to the WG (weight at 6 weeks of life, absolute WG, and WG proportion from birth to 6 weeks of life) achieved statistical significance after univariate analysis. The area under the ROC curve in our study was 0.63 (95\%CI: $0.495-0.761 ; P=0.037)$. For the discriminative cutoff of $51.2 \%$ of the WG proportion, sensitivity and specificity values were $66.3 \%$ and $62.6 \%$ respectively. Positive and negative predictive values were $10.2 \%$ and $94.7 \%$ respectively, thus meaning that a preterm who has gained more than $51.2 \%$ of weight proportional to his $\mathrm{BW}$, at the end of the sixth week of life, has around $94.7 \%$ chance of not developing severe ROP.

\section{Conclusions}

Our results have clinical importance because low WG, as defined and measured at the end of 6 weeks of life, was an easy identifiable risk factor, and helpful for predicting severe ROP some weeks in advance of the development of treatable ROP. In this way, ophthalmologists and neonatologists should take special care, and pay attention to this group of patients during the screening for ROP. There is a need for more studies assessing improvements in nutrition among VLBW preterm babies at risk for ROP to better understand this factor in ROP development.

Acknowledgement Authors wish to acknowledge Wolfgang William Schmidt Aguiar and Daniela Benzano for suggestions and orientations concerning the statistical analysis of this article.

Open Access This article is distributed under the terms of the Creative Commons Attribution Noncommercial License which permits any noncommercial use, distribution, and reproduction in any medium, provided the original author(s) and source are credited.

\section{References}

1. Terry T (1942) Extreme prematurity and fibroblastic overgrowth of persistent vascular sheath behind each crystalline lens. I - Preliminary report. Am J Ophthalmol 25:203-204

2. Gilbert C, Rahi J, Eckstein M, O’Sullivan J, Foster A (1997) Retinopathy of prematurity in middle-income countries. Lancet 350:12-14. doi:10.1016/S0140-6736(97)01107-0

3. Gilbert C, Fielder A, Gordillo L, Quinn G, Semiglia R, Visintin P et al (2005) Characteristics of infants with severe retinopathy of prematurity in countries with low, moderate, and high levels of development: implications for screening programs. Pediatrics 115 : e518-e525, doi:10.1542/peds.2004-1180

4. Shah VA, Yeo CL, Ling YL, Ho LY (2005) Incidence, risk factors of retinopathy of prematurity among very low birth weight infants in Singapore. Ann Acad Med Singapore 34:169-178

5. Kim TI, Sohn J, Pi SY, Yoon YH (2004) Postnatal risk factors of retinopathy of prematurity. Paediatr Perinat Epidemiol 18:130 134, doi:10.1111/j.1365-3016.2003.00545.x

6. Brown MS, Baron AE, France EK, Hamman RF (2006) Association between higher cumulative doses of recombinant erythropoietin and risk for retinopathy of prematurity. J AAPOS 10:143-149, doi:10.1016/j.jaapos.2005.09.005

7. Wallace DK, Kylstra JA, Phillips SJ, Hall JG (2000) Poor postnatal weight gain: a risk factor for severe retinopathy of prematurity. J AAPOS 4:343-347, doi:10.1067/mpa.2000.110342

8. Allegaert K, Vanhole C, Casteels I, Naulaers G, Debeer A, Cossey $\mathrm{V}$ et al (2003) Perinatal growth characteristics and associated risk of developing threshold retinopathy of prematurity. J AAPOS 7:34-37, doi:10.1016/S1091-8531(02)42015-0

9. Löfqvist C, Andersson E, Sigurdsson J, Engström E, Hard A, Niklasson A et al (2006) Longitudinal postnatal weight and insulin-like growth factor I measurements in the prediction of retinopathy of prematurity. Arch Ophthalmol 124:1711-1718, doi:10.1001/archopht.124.12.1711

10. The Committee for the Classification of Retinopathy of Prematurity (1984) An international classification of retinopathy of prematurity. Arch Ophthalmol 102:1130-1134

11. The International Committee for the Classification of the Late Stages of Retinopathy of Prematurity (1987) An international classification of retinopathy of prematurity. II. The classification of retinal detachment. Arch Ophthalmol 105:906-912

12. Cryotherapy for Retinopathy of Prematurity Cooperative Group (1988) Multicenter trial of cryotherapy for retinopathy of prematurity. Preliminary results. Arch Ophthalmol 106:471-479

13. Ehrenkranz RA, Younes N, Lemons JA, Fanaroff AA, Donovan EF, Wright LL et al (1999) Longitudinal growth of hospitalized very low birth weight infants. Pediatrics 104:280-289, doi:10.1542/peds.104.2.280 
14. Patz A, Hoeck LE, de la Cruz E (1952) Studies on the effect of high oxygen administration in retrolental fibroplasia. 1. Nursery observations. Am J Ophthalmol 35:1248-1253

15. Saito Y, Omoto T, Cho Y, Hatsukawa Y, Fujimura M, Takeuchi T (1993) The progression of retinopathy of prematurity and fluctuation in blood gas tension. Graefes Arch Clin Exp Ophthalmol 231:151-156, doi:10.1007/BF00920938

16. Lucey JF, Dangman B (1984) A reexamination of the role of oxygen in retrolental fibroplasia. Pediatrics 73:82-96

17. Tin W, Wariyar U (2002) Giving small babies oxygen: 50 years of uncertainty. Semin Neonatol 7:361-367, doi:10.1053/siny.2002. 0130

18. Larsson E, Carle-Petrelius B, Cernerud G, Ots L, Wallin A, Holmström G (2002) Incidence of ROP in two consecutive Swedish population based studies. Br J Ophthalmol 86:11221126, doi:10.1136/bjo.86.10.1122

19. Hussain N, Clive J, Bhandari V (1999) Current incidence of retinopathy of prematurity, 1989-1997. Pediatrics 104:e26, doi:10.1542/peds.104.3.e26

20. Graziano RM, Leone CR, Cunha SL, Pinheiro AC (1997) Prevalence of retinopathy of prematurity in very low birth weight infants. J Pediatr (Rio J) 73:377-382

21. Procianoy RS, Garcia-Prats JA, Hittner HM, Adams JM, Rudolph AJ (1981) An association between retinopathy of prematurity and intraventricular hemorrhage in very low birth weight infants. Acta Paediatr Scand 70:473-477, doi:10.1111/j.1651-2227.1981.tb05725.x

22. Christiansen SP, Fray KJ, Spencer T (2002) Ocular outcomes in low birth weight premature infants with intraventricular hemorrhage. J Pediatr Ophthalmol Strabismus 39:157-165
23. Seiberth V, Linderkamp O (2000) Risk factors in retinopathy of prematurity. a multivariate statistical analysis. Ophthalmologica 214:131-135, doi:10.1159/000027482

24. Lermann VL, Fortes Filho JB, Procianoy RS (2006) The prevalence of retinopathy of prematurity in very low birth weight newborn infants. J Pediatr (Rio J) 82:27-32, doi:10.2223/JPED.1433

25. Koerner F, Bossi E, Wetzel C, Flury B (1986) Retinopathy of prematurity: the influence of gestational age and retinal maturity on the statistical behavior of risk factors. Graefes Arch Clin Exp Ophthalmol 224:40-45, doi:10.1007/BF02144132

26. Hall JG, Freedman SF, Kylstra JA (1995) Clinical course and systemic correlates of retinopathy of prematurity in quintuplets. Am J Ophthalmol 119:658-660

27. Holmes JM, Duffner LA (1996) The effect of postnatal growth retardation on abnormal neovascularization in the oxygen exposed neonatal rat. Curr Eye Res 15:403-409, doi:10.3109/02713689 608995831

28. Shaffer SG, Quimiro CL, Anderson JB, Hall RT (1987) Postnatal weight changes in low birth weight infants. Pediatrics 79:702

29. Hellström A, Engström E, Hard AL, Albertsson-Wikland K, Carlsson B, Niklasson A et al (2003) Postnatal serum insulin-like growth factor I deficiency is associated with retinopathy of prematurity and other complications of premature birth. Pediatrics 112:1016-1020, doi:10.1542/peds.112.5.1016

30. Engström E, Niklasson A, Wikland KA, Ewald U, Hellström A (2005) The role of maternal factors, postnatal nutrition, weight gain, and gender in regulation of serum IGF-I among preterm infants. Pediatr Res 57:605-610, doi:10.1203/01.PDR.0000155950. 67503.BC 